\title{
Multi-bump type nodal solutions having a prescribed number of nodal domains: I
}

\section{Solutions nodales de multi-bosse ayant un nombre de domaines nodales prescrites: I}

\author{
Zhaoli Liu $^{\mathrm{a}, *, 1}$, Zhi-Qiang Wang ${ }^{\mathrm{b}}$ \\ a Department of Mathematics, Capital Normal University, Beijing 100037, PR China \\ ${ }^{\mathrm{b}}$ Department of Mathematics and Statistics, Utah State University, Logan, Utah 84322, USA
}

Received 16 January 2004; accepted 6 October 2004

Available online 7 April 2005

\begin{abstract}
This paper is concerned with analyzing their nodal property of multi-bump type sign-changing solutions constructed by Coti Zelati and Rabinowitz [Comm. Pure Appl. Math. 45 (1992) 1217]. In some cases we provide both upper and lower bounds on the number of nodal domains for these solutions.

(C) 2005 L'Association Publications de l'Institut Henri Poincaré. Published by Elsevier B.V. All rights reserved

\section{Résumé}

Cet article concerne l'analyse de la propriété nodale des solutions de multi-bosse changeant de signe, construites par Coti Zelati et Rabinowitz [Comm. Pure Appl. Math. 45 (1992) 1217]. Dans certain cas, nous obtenons la borne supérieure et inférieure de nombre des domaines nodales de ces solutions.

(C) 2005 L'Association Publications de l'Institut Henri Poincaré. Published by Elsevier B.V. All rights reserved
\end{abstract}

MSC: 35B05; $35 \mathrm{~J} 60$

Keywords: Semilinear elliptic equation; Multi-bump nodal solution; Number of nodal domains

\footnotetext{
* Corresponding author.

E-mail address: zliu@mail.cnu.edu.cdu (Z. Liu).

1 Supported by NSFC: 10441003.
}

0294-1449/\$ - see front matter (C) 2005 L'Association Publications de l'Institut Henri Poincaré. Published by Elsevier B.V. All rights reserved doi:10.1016/j.anihpc.2004.10.002 


\section{Introduction}

This paper is concerned with constructing multi-bump type, nodal (sign-changing) solutions which have a prescribed number of nodal domains for nonlinear time-independent Schrödinger equations of the form

$$
-\Delta u+V(x) u=f(x, u) \quad \text { in } \Omega, \quad u=0 \quad \text { on } \partial \Omega,
$$

which satisfy $u(x) \rightarrow 0$ as $|x| \rightarrow \infty$, here $\Omega$ is a smooth domain in $\mathbf{R}^{N}$ or the whole space $\mathbf{R}^{N}$. This type of equations arise from study of steady state and standing wave solutions of time-dependent nonlinear Schrödinger equations. If $u \in C\left(\mathbf{R}^{N}, \mathbf{R}\right)$ is a solution, each connected component of the set $\mathbf{R}^{N} \backslash u^{-1}(0)$ is called a nodal domain of $u$. The potential function is assumed to be periodic in the unbounded directions of $\Omega$. Multi-bump type solutions for nonlinear elliptic PDEs with a periodic potential was first obtained by Coti Zelati and Rabinowitz [11] by using a gluing method which was used initially for Hamiltonian ODEs in [10,22] (see the survey monographs of Rabinowitz [19-21] for more references therein). The gluing procedure is to use a variational argument to find a family of ground state solutions which constitute the basic 'one-bump' solutions. These one-bump solutions need to have a certain non-degeneracy property. Then further variational arguments are used to construct multi-bump solutions, i.e. solutions near sums of sufficiently separated translates of the basic solutions. In [11] the basic building blocks, one-bump solutions, are allowed to be either positive and negative ground state solutions. Thus multi-bump type nodal solutions potentially having many nodal domains are constructed. However, the question on the exact number of nodal domains for these multi-bump solutions was left open. A related question is to provide estimates on the number of nodal domains in terms of the minimax gluing procedure. To our knowledge this question has not been addressed either except in [1] nodal solutions having exactly two nodal domains were claimed. In this paper we shall partially address these questions. Another motivation for our study is that in recent years, nodal solutions of nonlinear elliptic BVPs have received much attention (e.g. [2-5,7,12,13,15-17]) in which many multiplicity results of nodal solutions were given both for bounded domains and for entire space. However, without any symmetry of subgroups of $\mathcal{O}(N)$ for the equations and the domains and without assuming the nonlinearity being odd in $u$, it is only known that there are nodal solutions having exactly two nodal domains. There seem no examples giving nodal solutions of more than two nodal domains, let alone giving a prescribed large number of nodal domains. We shall give results in this paper that claim the existence of nodal solutions having an arbitrary prescribed number of nodal domains. We shall build upon the ideas and approaches used by Coti Zelati-Rabinowitz [11] and exploit further finer estimates of the multi-bump type solutions so that their nodal property can be analyzed qualitatively.

If $\Omega$ is the entire $\mathbf{R}^{N}$, we rewrite Eq. (1) as

$$
-\Delta u+V(x) u=f(x, u), \quad \text { in } \mathbf{R}^{N} .
$$

Depending on whether $\Omega$ is a cylindrical domain or the entire $\mathbf{R}^{N}$ and on whether $V$ and $f$ are periodic in all $x$ variables, we shall consider three different cases.

Case (i). Our first result is for Eq. (2). We make the following assumptions on $V$ and $f$ :

$\left(\mathrm{V}_{1}\right) \quad V \in C\left(\mathbf{R}^{N}, \mathbf{R}\right), V_{0}=\inf _{\mathbf{R}^{N}} V(x)>0$, is periodic in $x_{1}, \ldots, x_{N}$.

(f $\left.\mathrm{f}_{1}\right) f \in C^{1}\left(\mathbf{R}^{N} \times \mathbf{R}, \mathbf{R}\right)$ is periodic in $x_{1}, \ldots, x_{N}$.

(f $\left.f_{2}\right) f(x, 0)=0=f_{u}(x, 0)$.

(f $\left.\mathrm{f}_{3}\right)$ There is $C>0$ such that

$$
\left|f_{u}(x, u)\right| \leqslant C\left(1+|u|^{p-2}\right)
$$

for all $x \in \mathbf{R}^{N}, u \in \mathbf{R}$ where $2<p<2^{*}$.

( $\left.\mathrm{f}_{4}\right)$ There is $\mu>2$ such that

$$
0<\mu F(x, u):=\mu \int_{0}^{u} f(x, t) \mathrm{d} t \leqslant u f(x, u)
$$


for all $x \in \mathbf{R}^{N}, u \in \mathbf{R} \backslash\{0\}$.

(f 5 ) $f(x,-u)=-f(x, u)$, i.e., $f$ is odd in $u$.

The weak solutions of (2) correspond to critical points of

$$
I(u):=\frac{1}{2} \int_{\mathbf{R}^{N}}\left(|\nabla u|^{2}+V(x) u^{2}\right) \mathrm{d} x-\int_{\mathbf{R}^{N}} F(x, u) \mathrm{d} x,
$$

in $E=W^{1,2}\left(\mathbf{R}^{N}\right)$. We shall use the following notations as in [10,11]. $I^{b}=\{u \in E \mid I(u) \leqslant b\}, I_{a}=\{u \in E \mid I(u) \geqslant$ $a\}, I_{a}^{b}=\{u \in E \mid a \leqslant I(u) \leqslant b\}, \mathcal{K}=\left\{u \in E \mid I^{\prime}(u)=0\right\}, \mathcal{K}(c)=\left\{u \in E \mid I^{\prime}(u)=0, I(u)=c\right\}, \mathcal{K}^{b}=\mathcal{K} \cap I^{b}$, $\mathcal{K}_{a}^{b}=\mathcal{K} \cap I_{a}^{b}$.

Due to the periodicity in the $x_{1}, \ldots, x_{N}$ directions the problem is invariant under translations in the $x_{1}, \ldots, x_{N}$ directions, i.e., being $\mathbf{Z}^{N}$-invariant. With the superlinear nonlinearity $f(x, u)$ we may define the mountain pass value $c>0$.

$$
c=\inf _{g \in \Gamma} \sup _{t \in[0,1]} I(g(t))
$$

where

$$
\Gamma=\left\{g \in C([0,1], E) \mid g(0)=0, g(1) \in I^{0} \backslash\{0\}\right\} .
$$

As in $[10,11]$, we assume

(*) There is $\alpha>0$ such that $\mathcal{K}^{c+\alpha} / \mathbf{Z}^{N}$ is finite.

According to [11], the above assumptions guarantee the existence of positive and negative solutions to Eq. (2) at the mountain-pass level. These solutions will be referred to as one-bump solutions. Let $v_{1}, \ldots, v_{k}$ be one-bump solutions such that their barycenters are sufficiently separated. Solutions of (2) that are close to $\sum_{i=1}^{k} v_{i}$ are called $k$-bump solutions. We understand this reference also applies to Eq. (1).

Theorem 1.1. Assume $\left(\mathrm{V}_{1}\right)$ and $\left(\mathrm{f}_{1}\right)-\left(\mathrm{f}_{5}\right)$. Suppose $(*)$ holds. For multi-bump nodal solutions of Eq. (2), the number of nodal domains is bounded by the number of bumps. In particular, the two-bump nodal solutions have exactly two nodal domains. Moreover, there are infinitely many, geometrically different, two-bump, nodal solutions which have exactly two nodal domains.

Case (ii). Next we consider Eq. (1) on a cylinder domain $\Omega=\omega \times \mathbf{R}$ and we write $x=\left(x^{\prime}, x_{N}\right)$ with $x^{\prime}=$ $\left(x_{1}, \ldots, x_{N-1}\right)$, where $\omega$ is a bounded smooth domain in $\mathbf{R}^{N-1}$. We assume that

$\left(\mathrm{V}_{1^{\prime}}\right) V \in C(\Omega, \mathbf{R}), V_{0}:=\inf _{\Omega} V(x)>0$, is periodic in $x_{N}$.

$\left(\mathrm{f}_{1^{\prime}}\right) f \in C^{1}(\Omega \times \mathbf{R}, \mathbf{R})$ is periodic in $x_{N}$.

We understand $\left(\mathrm{f}_{2}\right)-\left(\mathrm{f}_{5}\right)$ are satisfied now for $x \in \Omega$. The weak solutions of (1) correspond to critical points of

$$
I(u):=\frac{1}{2} \int_{\Omega}\left(|\nabla u|^{2}+V(x) u^{2}\right) \mathrm{d} x-\int_{\Omega} F(x, u) \mathrm{d} x,
$$

in $E=H_{0}^{1}(\Omega)$. Then we can still define the mountain pass value $c>0$. The problem now is $\mathbf{Z}$ invariant.

$\left(*^{\prime}\right)$ There is $\alpha>0$ such that $\mathcal{K}^{c+\alpha} / \mathbf{Z}$ is finite. 
Theorem 1.2. Assume $\left(\mathrm{V}_{1^{\prime}}\right),\left(\mathrm{f}_{1^{\prime}}\right)$, and $\left(\mathrm{f}_{2}\right)-\left(\mathrm{f}_{5}\right)$. Suppose $\left(*^{\prime}\right)$ holds. Then for any integers $k \geqslant m \geqslant 2$, Eq. (1) has infinitely many, geometrically different, $k$-bump, nodal solutions in $I_{k c-\alpha}^{k c+\alpha}$ which have exactly $m$ nodal domains. More precisely, given any positive integers $k_{1}, k_{2}, \ldots, k_{m}$ such that $\sum_{i=1}^{m} k_{i}=k \geqslant 2$, there are infinitely many, geometrically different, $k$-bump, nodal solutions in $I_{k c-\alpha}^{k c+\alpha}$ which have exactly $m$ nodal domains $D_{i}, i=1, \ldots, m$, such that $\left.u\right|_{D_{i}}$ is a $k_{i}$-bump positive or negative solution.

Case (iii). Finally, we consider Eq. (2) with different $x$-dependence in different directions.

$\left(\mathrm{V}_{1^{\prime \prime}}\right) V \in C\left(\mathbf{R}^{N}, \mathbf{R}\right), V_{0}:=\inf _{\mathbf{R}^{N}} V(x)>0$, is periodic in $x_{N}$ and radially symmetric in $\left(x_{1}, \ldots, x_{N-1}\right)$.

$\left(\mathrm{f}_{1^{\prime \prime}}\right) f \in C^{1}\left(\mathbf{R}^{N} \times \mathbf{R}, \mathbf{R}\right)$ is periodic in $x_{N}$ and radially symmetric in $\left(x_{1}, \ldots, x_{N-1}\right)$.

Then the problem is again $\mathbf{Z}$-invariant. With $x=\left(x^{\prime}, x_{N}\right)$ and $x^{\prime}=\left(x_{1}, \ldots, x_{N-1}\right)$, we take

$$
E=\left\{u \in W^{1,2}\left(\mathbf{R}^{N}\right) \mid u\left(x^{\prime}, x_{N}\right)=u\left(\left|x^{\prime}\right|, x_{N}\right), \int_{\mathbf{R}^{N}} V(x) u^{2} \mathrm{~d} x<\infty\right\}
$$

i.e., functions in $E$ are radially symmetric in the first $N-1$ variables. We can still define the mountain pass value $c$ in the space $E$.

$\left(*^{\prime \prime}\right)$ There is $\alpha>0$ such that $\mathcal{K}^{c+\alpha} / \mathbf{Z}$ is finite.

Theorem 1.3. Assume $\left(\mathrm{V}_{1^{\prime \prime}}\right),\left(\mathrm{f}_{1^{\prime \prime}}\right)$ and $\left(\mathrm{f}_{2}\right)-\left(\mathrm{f}_{5}\right)$. Suppose $\left(*^{\prime \prime}\right)$ holds. For any integer $k \geqslant 2$, Eq. $(2)$ has infinitely many, geometrically different, $k$-bump, nodal solutions in $I_{k c-\alpha}^{k c+\alpha}$ such that the numbers of their nodal domains are bounded between $[k / 2]+1$ and $k$. In particular, there are nodal solutions such that the numbers of their nodal domains tend to infinity.

Remark 1.4. In the setting of Theorem 1.2, one can easily see that $\Omega$ can be taken as more general smooth domain than a standard cylinder domain in $\mathbf{R}^{N}$, which needs to be periodic in the $x_{N}$ direction and whose projection on $\mathbf{R}^{N-1}$ is bounded. Furthermore, it follows from the proof that this $(N-1)$-dimensional projection may also be unbounded. In this case, we need to assume that there is a number $t$ such that $\left\{x^{\prime} \in \mathbf{R}^{N-1} \mid\left(x^{\prime}, t\right) \in \Omega\right\}$ is bounded and impose additional assumptions on $V$ and $f$ in $\mathbf{R}^{N-1}$ direction, for example, $V$ is coercive in the $(N-1)$ space. Finally, in the case the equation is autonomous, namely, independent of $x$, we may assume the domains are periodic in the $x_{N}$ direction. By assuming an isolatedness condition of the critical points at the mountain pass level like $\left(*^{\prime}\right)$, a similar result to Theorem 1.2 can be stated.

Remark 1.5. The assumption that $f$ is odd in $u$ is a technical condition but used in [11] in an essential way. In a sequel to this paper [18] we will provide techniques to remove this condition. This requires modifications of the gluing procedure of [11] by combining with invariant set method.

The paper is organized as follows. Section 2 contains a sketch of the original construction of multi-bump solutions in the setting of Theorem 1.1 due to Coti Zelati-Rabinowitz [11], and some variants of it for the settings of our Theorems 1.2 and 1.3. Section 3 is devoted to the proof of our main estimates about $C^{1}$-closeness of the multi-bump solutions to the sum of the basic one bump solutions. In Section 4, using the results from Section 3 we prove our main Theorems 1.1, 1.2, and 1.3. 


\section{Multi-bump type nodal solutions revisited}

In this section $E$ denotes the Sobolev space $W^{1,2}\left(\mathbf{R}^{N}\right)$, and some times a subspace of it when the context is clear.

We consider the case (i) (Theorem 1.1) first which was considered in [11] in detail by Coti Zelati-Rabinowitz. Without loss of generality we assume the periods in all directions are equal to 1 .

Let $j=\left(j_{1}, \ldots, j_{N}\right) \in \mathbf{Z}^{N}$ and the translations on the $\mathbf{R}^{N}$ will be defined by

$$
\tau_{j} u(x)=u\left(x_{1}+j_{1}, \ldots, x_{N}+j_{N}\right) .
$$

Following [11], one can prove that there is $v>0$ such that for all $v \in \mathcal{K} \backslash\{0\},\|v\| \geqslant v$. Under (*), it is proved in [11] that the mountain pass value $c$ is a critical value. Let $\Lambda=\{+1,-1\}$ and $\lambda=\left(\lambda_{1}, \ldots, \lambda_{k}\right) \in \Lambda^{k}$. Then one chooses a finite set $A \subset \mathcal{K}(c)$ which contains only positive solutions (see [11] for the details) to define for any fixed integer $k \geqslant 2, \lambda=\left(\lambda_{1}, \ldots, \lambda_{k}\right) \in \Lambda^{k}$,

$$
\mathcal{M}=\mathcal{M}\left(j_{1}, \ldots, j_{k}, A, \lambda\right)=\left\{\sum_{i=1}^{k} \lambda_{i} \tau_{j_{i}} v_{i} \mid v_{i} \in A\right\},
$$

where $j_{i} \in \mathbf{Z}^{N}$ for $i=1, \ldots, k$ are fixed such that $\left\|\sum_{i=1}^{k} \tau_{j_{i}} v_{i}\right\| \geqslant \frac{k v}{2}$.

Theorem 2.1 [11]. Assume $\left(\mathrm{V}_{1}\right)$ and $\left(\mathrm{f}_{1}\right)-\left(\mathrm{f}_{5}\right)$. Suppose $(*)$ holds. There is $r_{0}>0$ such that for any $r \in\left(0, r_{0}\right)$

$$
N_{r}\left(\mathcal{M}\left(l_{j_{1}}, \ldots, l j_{k}, A, \lambda\right)\right) \cap\left(\mathcal{K}_{k c-\alpha}^{k c+\alpha} / \mathbf{Z}^{N}\right) \neq \varnothing
$$

for all but finitely many $l \in \mathbf{N}$, where $N_{r}(\cdot)$ is the $r$-neighborhood in $E$.

Next consider case (ii). In the setting of Theorem 1.1 which was sketched in [11] we state the following result. Here the problem is only $\mathbf{Z}$-invariant and we understand $j \in \mathbf{Z}$ here and the translations $\tau_{j}$ are only in the $x_{N}$ direction.

Theorem 2.2. Assume $\left(\mathrm{V}_{1^{\prime}}\right),\left(\mathrm{f}_{1^{\prime}}\right)$, and $\left(\mathrm{f}_{2}\right)-\left(\mathrm{f}_{5}\right)$. Suppose $\left(*^{\prime}\right)$ holds. There is $r_{0}>0$ such that for any $r \in\left(0, r_{0}\right)$

$$
N_{r}\left(\mathcal{M}\left(l_{j_{1}}, \ldots, l_{j_{k}}, A, \lambda\right)\right) \cap\left(\mathcal{K}_{k c-\alpha}^{k c+\alpha} / \mathbf{Z}\right) \neq \emptyset
$$

for all but finitely many $l \in \mathbf{N}$, where $N_{r}(\cdot)$ is the $r$-neighborhood in $E=W_{0}^{1,2}(\Omega)$.

Though this result was not stated in [11], the multi-bump solutions without nodal information were stated there. With the modifications for the case of Theorem 2.1 little needs to be added for getting Theorem 2.2.

Finally we consider the case of Theorem 1.3 corresponding to case (iii). We note again the space $E$ we employ here is a subspace of $W^{1,2}\left(\mathbf{R}^{N}\right)$ which contains functions radially symmetric with respect to the first $(N-1)$ variables.

Theorem 2.3. Assume $\left(\mathrm{V}_{1^{\prime \prime}}\right),\left(\mathrm{f}_{1^{\prime \prime}}\right)$, and $\left(\mathrm{f}_{2}\right)-\left(\mathrm{f}_{5}\right)$. Suppose $\left(*^{\prime \prime}\right)$ holds. There is $r_{0}>0$ such that for any $r \in\left(0, r_{0}\right)$

$$
N_{r}\left(\mathcal{M}\left(l_{j_{1}}, \ldots, l j_{k}, A, \lambda\right)\right) \cap\left(\mathcal{K}_{k c-\alpha}^{k c+\alpha} / \mathbf{Z}\right) \neq \emptyset
$$

for all but finitely many $l \in \mathbf{N}$, where $N_{r}(\cdot)$ is the $r$-neighborhood in $E$.

To establish this result we note that checking through the arguments in [11] for proving Theorem 2.1 all the procedures used in [11] can be confined in the subspace $E$ in our case by taking care of the symmetry in the first $(N-1)$ variables. We omit the proofs here. Theorem 2.3 is also valid if $V$ and $f$ are radially symmetric in $x_{1}, \ldots, x_{n}$ and periodic in $x_{n+1}, \ldots, x_{N}$ for some $1<n<N$. 
By using the arguments in Proposition 7.32 of [11], one can prove that if $\lambda \neq(+1, \ldots,+1)$ or $(-1, \ldots,-1)$, and for $r$ sufficiently small and $l$ sufficiently large, the solutions given in the above three theorems are nodal (sign-changing) solutions.

Next we state a result which is one of the main ingredients in establishing more detailed nodal property of these solutions.

Theorem 2.4. In the above three theorems, if $r$ is sufficiently small and $l$ sufficiently large, we may replace the $r$ neighborhood in $E$ with $r$-neighborhood in $X$ where $X=C^{1}(\bar{\Omega})$ in Theorem 2.2 and $X=C^{1}\left(\mathbf{R}^{N}\right)$ in Theorems 2.1 and 2.3.

Remark 2.5. Theorem 2.4 says the multi-bump solutions constructed in [11] are close to the sum of translates of the one bump basic solutions not only in the Sobolev norm but also in the stronger $C^{1}$ norm. This in some sense resembles Brezis and Nirenberg's remarks on minimizers [6] as well as the remarks by Chang on minimax critical points $[8,9]$ and suggests that the minimax procedure of Coti Zelati and Rabinowitz [11] can be posed in the $C^{1}$ topology where the multi-bump feature is more apparent.

The proof of this result is given in Section 3. Using it and some maximum principle arguments we prove Theorems 1.1, 1.2 and 1.3 in Section 4.

\section{3. $C^{1}$-estimates}

In this section, we will prove Theorem 2.4. It suffices to prove the following lemma.

Lemma 3.1. Let $l_{n} \rightarrow \infty$ as $n \rightarrow \infty$ and $u_{n} \in \mathcal{K}_{k c-\alpha}^{k c+\alpha}$ such that

$$
\lim _{n \rightarrow \infty} \operatorname{dist}_{E}\left(u_{n}, \mathcal{M}\left(l_{n} j_{1}, \ldots, l_{n} j_{k}, A, \lambda\right)\right)=0 .
$$

Then

$$
\lim _{n \rightarrow \infty} \operatorname{dist}_{X}\left(u_{n}, \mathcal{M}\left(l_{n} j_{1}, \ldots, l_{n} j_{k}, A, \lambda\right)\right)=0 .
$$

Proof. We will only prove the result in the case of $\mathbf{R}^{N}$. The other case can be treated similarly. Since $A$ is a finite set, without loss of generality we may assume that as $n \rightarrow \infty$

$$
\left\|u_{n}-\sum_{i=1}^{k} \lambda_{i} \tau_{l_{n} j_{i}} v_{i}\right\| \rightarrow 0
$$

for some $\left\{v_{1}, \ldots, v_{k}\right\} \subset A$. Denote for $n=1,2, \ldots, w_{n}=u_{n}-\sum_{i=1}^{k} \lambda_{i} \tau_{l_{n} j_{i}} v_{i}$. Since

$$
-\Delta\left(\lambda_{i} \tau_{l_{n} j_{i}} v_{i}\right)+V(x)\left(\lambda_{i} \tau_{l_{n} j_{i}} v_{i}\right)=f\left(x, \lambda_{i} \tau_{l_{n} j_{i}} v_{i}\right)
$$

and

$$
-\Delta u_{n}+V(x) u_{n}=f\left(x, u_{n}\right),
$$

$w_{n}$ satisfies

$$
-\Delta w_{n}+V(x) w_{n}=f\left(x, u_{n}\right)-\sum_{i=1}^{k} f\left(x, \lambda_{i} \tau_{l_{n} j_{i}} v_{i}\right)=z_{n}^{(1)}+z_{n}^{(2)},
$$


where

$$
z_{n}^{(1)}:=f\left(x, u_{n}\right)-f\left(x, \sum_{i=1}^{k} \lambda_{i} \tau_{l_{n} j_{i}} v_{i}\right)=\int_{0}^{1} f_{u}\left(x, \sum_{i=1}^{k} \lambda_{i} \tau_{l_{n} j_{i}} v_{i}+s w_{n}\right) \mathrm{d} s \cdot w_{n}
$$

and

$$
z_{n}^{(2)}:=f\left(x, \sum_{i=1}^{k} \lambda_{i} \tau_{l_{n} j_{i}} v_{i}\right)-\sum_{i=1}^{k} f\left(x, \lambda_{i} \tau_{l_{n} j_{i}} v_{i}\right)
$$

The rest of the proof will be divided into four steps.

Step 1: For any $r>2, \int_{\mathbf{R}^{N}}\left|w_{n}\right|^{r} \mathrm{~d} x \rightarrow 0$ as $n \rightarrow \infty$. With $V_{0}=\inf V(x)>0$, by $\left(\mathrm{f}_{2}\right)$ and (f $\left.\mathrm{f}_{3}\right)$, for all $x \in \mathbf{R}^{N}$ and $u \in \mathbf{R}$,

$$
\left|f_{u}(x, u)\right| \leqslant \frac{V_{0}}{2}+C_{1}|u|^{p-2} .
$$

Here and in the sequel, $C_{i}$ stands for positive constants independent of $n$ and $x$. For any $\alpha>0$, multiplying (3) with $\left|w_{n}\right|^{\alpha} w_{n}$ and integrating over $\mathbf{R}^{N}$, we have

$$
\int_{\mathbf{R}^{N}}\left(\left|\nabla\left(\left|w_{n}\right|^{\alpha / 2} w_{n}\right)\right|^{2}+V(x)\left|w_{n}\right|^{\alpha+2}\right) \mathrm{d} x \leqslant C_{2} \int_{\mathbf{R}^{N}}\left(\left|z_{n}^{(1)}\right|+\left|z_{n}^{(2)}\right|\right)\left|w_{n}\right|^{\alpha+1} \mathrm{~d} x .
$$

Since (4) and (6) imply

$$
\left|z_{n}^{(1)}\right| \leqslant \frac{V_{0}}{2}\left|w_{n}\right|+C_{3}\left(\left|u_{n}\right|^{p-2}+\sum_{i=1}^{k}\left|\tau_{l_{n} j_{i}} v_{i}\right|^{p-2}\right)\left|w_{n}\right|,
$$

it follows from (7) that

$$
\begin{aligned}
\int_{\mathbf{R}^{N}}\left(\left|\nabla\left(\left|w_{n}\right|^{\alpha / 2} w_{n}\right)\right|^{2}+\left(V(x)-\frac{V_{0}}{2}\right)\left|w_{n}\right|^{\alpha+2}\right) \mathrm{d} x \\
\leqslant C_{4}(\alpha)\left[\left(\int_{\mathbf{R}^{N}}\left|u_{n}\right|^{2^{*}} \mathrm{~d} x\right)^{\frac{p-2}{2^{*}}}+\sum_{i=1}^{k}\left(\int_{\mathbf{R}^{N}}\left|v_{i}\right|^{2^{*}} \mathrm{~d} x\right)^{\frac{p-2}{2^{*}}}\right]\left(\int_{\mathbf{R}^{N}}\left|w_{n}\right|^{\frac{(\alpha+2) 2^{*}}{2^{*}-p+2}} \mathrm{~d} x\right)^{\frac{2^{*}-p+2}{2^{*}}} \\
+\left(\int_{\mathbf{R}^{N}}\left|z_{n}^{(2)}\right|^{\frac{(\alpha+2) 2^{*}}{2^{*}+(\alpha+1)(p-2)}} \mathrm{d} x\right)^{\frac{2^{*}+(\alpha+1)(p-2)}{(\alpha+2) 2^{*}}}\left(\int_{\mathbf{R}^{N}}\left|w_{n}\right|^{\frac{(\alpha+2) 2^{*}}{2^{*}-p+2}} \mathrm{~d} x\right)^{\frac{(\alpha+1)\left(2^{*}-p+2\right)}{(\alpha+2) 2^{*}}}
\end{aligned}
$$

Noting that $\left\{\left\|u_{n}\right\|\right\}$ is bounded and $\left\{\left\|z_{n}^{(2)}\right\|_{L^{r}\left(\mathbf{R}^{N}\right)}\right\}$ has a bound independent of $n$ for any $r>2$ and using Sobolev inequality, we see that

$$
\left(\int_{\mathbf{R}^{N}}\left|w_{n}\right|^{\frac{(\alpha+2) 2^{*}}{2}} \mathrm{~d} x\right)^{\frac{2}{2^{*}}} \leqslant C_{5}(\alpha)\left[\left(\int_{\mathbf{R}^{N}}\left|w_{n}\right|^{\frac{(\alpha+2) 2^{*}}{2^{*}-p+2}} \mathrm{~d} x\right)^{\frac{2^{*}-p+2}{2^{*}}}+\left(\int_{\mathbf{R}^{N}}\left|w_{n}\right|^{\frac{(\alpha+2) 2^{*}}{2^{*}-p+2}} \mathrm{~d} x\right)^{\frac{(\alpha+1)\left(2^{*}-p+2\right)}{(\alpha+2) 2^{*}}}\right] .
$$

Choosing $\alpha=\alpha_{1}:=2^{*}-p$ in (8) and using Sobolev inequality again, we have

$$
\left(\int_{\mathbf{R}^{N}}\left|w_{n}\right|^{\frac{\left(2^{*}-p+2\right) 2^{*}}{2}} \mathrm{~d} x\right)^{\frac{2}{2^{*}}} \leqslant C_{6}\left(\left\|w_{n}\right\|^{2^{*}-p+2}+\left\|w_{n}\right\|^{\frac{\left(\alpha_{1}+1\right)\left(2^{*}-p+2\right)}{\alpha_{1}+2}}\right),
$$


while choosing $\alpha=\alpha_{m}:=2\left(\frac{2^{*}-p+2}{2}\right)^{m}-2$ in (8) yields for any positive integer $m$,

$$
\begin{aligned}
\left(\int_{\mathbf{R}^{N}}\left|w_{n}\right|^{\frac{\left(2^{*}-p+2\right)^{m} 2^{*}}{2^{m}}} \mathrm{~d} x\right)^{\frac{2}{2^{*}} \leqslant} \leqslant & C_{7}\left[\left(\int_{\mathbf{R}^{N}}\left|w_{n}\right|^{\frac{\left(2^{*}-p+2\right)^{m-1} 2^{*}}{2^{m-1}}} \mathrm{~d} x\right)^{\frac{2^{*}-p+2}{2^{*}}}\right. \\
& \left.+\left(\int_{\mathbf{R}^{N}}\left|w_{n}\right|^{\frac{\left(2^{*}-p+2\right)^{m-1} 2^{*}}{2^{m-1}}} \mathrm{~d} x\right)^{\frac{\left(\alpha_{m}+1\right)\left(2^{*}-p+2\right)}{\left(\alpha_{m}+2\right) 2^{*}}}\right] .
\end{aligned}
$$

Since $\left\|w_{n}\right\| \rightarrow 0$ as $n \rightarrow \infty$ and $\left(2^{*}-p+2\right)^{m} / 2^{m} \rightarrow \infty$ as $m \rightarrow \infty$, an iterative process based on (9) and (10) shows that $\int_{\mathbf{R}^{N}}\left|w_{n}\right|^{r} \mathrm{~d} x \rightarrow 0$ as $n \rightarrow \infty$ for any $r>2$.

Step 2: For any $r>2, \int_{\mathbf{R}^{N}}\left|z_{n}^{(1)}\right|^{r} \mathrm{~d} x \rightarrow 0$ as $n \rightarrow \infty$. Indeed, by (4) and ( $\left.\mathrm{f}_{3}\right)$,

$$
\left|z_{n}^{(1)}\right| \leqslant C_{8}\left(\left|w_{n}\right|+\left|w_{n}\right|^{p-1}\right) .
$$

Thus the result in step 1 implies that $\int_{\mathbf{R}^{N}}\left|z_{n}^{(1)}\right|^{r} \mathrm{~d} x \rightarrow 0$ for any $r>2$.

Step 3: For any $r>2, \int_{\mathbf{R}^{N}}\left|z_{n}^{(2)}\right|^{r} \mathrm{~d} x \rightarrow 0$ as $n \rightarrow \infty$. For any $\epsilon>0$, choose $R>0$ such that for all $i \in\{1, \ldots, k\}$,

$$
\int_{\mathbf{R}^{N} \backslash B_{R}(0)}\left|v_{i}\right|^{r} \mathrm{~d} x<\epsilon .
$$

Enlarging $R$ if necessary, we can also assume that $\left|v_{i}(x)\right|<1$ for all $i \in\{1, \ldots, k\}$ and $x \in \mathbf{R}^{N} \backslash B_{R}(0)$. Thus for $x \in G(R, n):=\mathbf{R}^{N} \backslash \bigcup_{i=1}^{k} B_{R}\left(l_{n} j_{i}\right)$,

$$
\left|\sum_{i=1}^{k} \lambda_{i} \tau_{l_{n} j_{i}} v_{i}\right|<k .
$$

Since $|f(x, u)| \leqslant C_{9}|u|$ for $|u| \leqslant k$, from (5) we see that

$$
\begin{aligned}
\int_{G(R, n)}\left|z_{n}^{(2)}\right|^{r} \mathrm{~d} x & =\int_{G(R, n)}\left|f\left(x, \sum_{i=1}^{k} \lambda_{i} \tau_{l_{n} j_{i}} v_{i}\right)-\sum_{i=1}^{k} f\left(x, \lambda_{i} \tau_{l_{n} j_{i}} v_{i}\right)\right|^{r} \mathrm{~d} x \\
& \leqslant C_{10} \sum_{i=1}^{k} \int_{G(R, n)}\left|\tau_{l_{n} j_{i}} v_{i}\right|^{r} \mathrm{~d} x .
\end{aligned}
$$

Combining (11) and (12) yields

$$
\int_{G(R, n)}\left|z_{n}^{(2)}\right|^{r} \mathrm{~d} x \leqslant C_{11} \epsilon .
$$

Choose $N_{0}$ such that if $n \geqslant N_{0}$ then $l_{n}\left|j_{i}-j_{m}\right|>2 R$ for any $i \neq m$. On each ball $B_{R}\left(l_{n} j_{m}\right)$ with $m \in\{1, \ldots, k\}$ and $n \geqslant N_{0}$, we have from (5) again

$$
\begin{gathered}
\int_{B_{R}\left(l_{n} j_{m}\right)}\left|z_{n}^{(2)}\right|^{r} \mathrm{~d} x \leqslant \\
2^{r} \int_{B_{R}\left(l_{n} j_{m}\right)}\left|f\left(x, \sum_{i=1}^{k} \lambda_{i} \tau_{l_{n} j_{i}} v_{i}\right)-f\left(x, \lambda_{m} \tau_{l_{n} j_{m}} v_{m}\right)\right|^{r} \mathrm{~d} x \\
+2^{r} \int_{B_{R}\left(l_{n} j_{m}\right)}\left|\sum_{i \neq m} f\left(x, \lambda_{i} \tau_{l_{n} j_{i}} v_{i}\right)\right|^{r} \mathrm{~d} x
\end{gathered}
$$




$$
\begin{aligned}
& \leqslant 2^{r} \int_{B_{R}\left(l_{n} j_{m}\right)}\left|\int_{0}^{1} f_{u}\left(x, \lambda_{m} \tau_{l_{n} j_{m}} v_{m}+s \sum_{i \neq m} \lambda_{i} \tau_{l_{n} j_{i}} v_{i}\right) \mathrm{d} s\right|\left|\sum_{i \neq m} \lambda_{i} \tau_{l_{n} j_{i}} v_{i}\right|^{r} \mathrm{~d} x \\
& +2^{r} \int_{B_{R}\left(l_{n} j_{m}\right)}\left|\sum_{i \neq m} f\left(x, \lambda_{i} \tau_{l_{n} j_{i}} v_{i}\right)\right|^{r} \mathrm{~d} x .
\end{aligned}
$$

Since for $u$ in a bounded interval, $f_{u}(x, u)$ is bounded and $|f(x, u)| \leqslant C_{12}|u|$, it follows that

$$
\int_{B_{R}\left(l_{n} j_{m}\right)}\left|z_{n}^{(2)}\right|^{r} \mathrm{~d} x \leqslant C_{13} \sum_{i \neq m} \int_{B_{R}\left(l_{n}\left(j_{m}-j_{i}\right)\right)}\left|v_{i}\right|^{r} \mathrm{~d} x .
$$

Then the fact that $B_{R}\left(l_{n}\left(j_{m}-j_{i}\right)\right) \cap B_{R}(0)=\emptyset$ and (11) imply that

$$
\sum_{m=1}^{k} \int_{B_{R}\left(l_{n} j_{m}\right)}\left|z_{n}^{(2)}\right|^{r} \mathrm{~d} x \leqslant C_{14} \epsilon .
$$

That $\int_{\mathbf{R}^{N}}\left|z_{n}^{(2)}\right|^{r} \mathrm{~d} x \rightarrow 0$ as $n \rightarrow \infty$ follows from (13) and (14).

Step 4: We complete the proof here. By the regularity theory of elliptic equations [14], for any $x \in \mathbf{R}^{N}$,

$$
\begin{aligned}
\left\|w_{n}\right\|_{W^{2,2 N}\left(B_{1}(x)\right)} & \leqslant C_{15}\left(\left\|w_{n}\right\|_{L^{2 N}\left(B_{2}(x)\right)}+\left\|z_{n}^{(1)}\right\|_{L^{2 N}\left(B_{2}(x)\right)}+\left\|z_{n}^{(2)}\right\|_{L^{2 N}\left(B_{2}(x)\right)}\right) \\
& \leqslant C_{15}\left(\left\|w_{n}\right\|_{L^{2 N}\left(\mathbf{R}^{N}\right)}+\left\|z_{n}^{(1)}\right\|_{L^{2 N}\left(\mathbf{R}^{N}\right)}+\left\|z_{n}^{(2)}\right\|_{L^{2 N}\left(\mathbf{R}^{N}\right)}\right) .
\end{aligned}
$$

By Sobolev inequality,

$$
\left\|w_{n}\right\|_{C^{1}\left(\overline{B_{1}(x)}\right)} \leqslant C_{16}\left\|w_{n}\right\|_{W^{2,2 N}\left(B_{1}(x)\right)} .
$$

Thus, since $x \in \mathbf{R}^{N}$ is arbitrary,

$$
\left\|w_{n}\right\|_{C^{1}\left(\mathbf{R}^{N}\right)} \leqslant C_{17}\left(\left\|w_{n}\right\|_{L^{2 N}\left(\mathbf{R}^{N}\right)}+\left\|z_{n}^{(1)}\right\|_{L^{2 N}\left(\mathbf{R}^{N}\right)}+\left\|z_{n}^{(2)}\right\|_{L^{2 N}\left(\mathbf{R}^{N}\right)}\right) .
$$

Then the results from steps $1-3$ imply

$$
\lim _{n \rightarrow \infty}\left\|w_{n}\right\|_{C^{1}\left(\mathbf{R}^{N}\right)}=0,
$$

finishing the proof.

We remark that the proof above does not use the condition of $f$ being odd.

\section{Proofs of the main results}

In the following we refer $k$ as the number of bumps of the solutions constructed in Section 2. The following lemma is true in all three cases we consider.

Lemma 4.1. If $r$ is small enough and $l$ is large enough then, for the solutions $u$ given in Section 2, the number of nodal domains is bounded above by the number of bumps of the solutions.

Proof. Let $\inf V(x) \geqslant V_{0}>0$. Then there is $\delta>0$ such that for $|t| \leqslant \delta,|f(x, t)| \leqslant V_{0}|t| / 2$. Since $A$ is finite there is $R_{0}>0$ such that $v(x) \leqslant \delta / 2 k$ for all $v \in A$ and $|x| \geqslant R_{0}$. In cases (i) and (iii), we have $\inf _{B_{R_{0}}}(0) v(x) \geqslant a_{0}>0$ for all $v \in A$ and for some $a_{0}>0$. Let $F_{l}=\bigcup_{i=1}^{k} B_{R_{0}}\left(l j_{i}\right)$. Suppose $u \in N_{r}\left(\mathcal{M}\left(l j_{1}, \ldots, l j_{k}, A, \lambda\right)\right) \cap \mathcal{K}_{k c-\alpha}^{k c+\alpha}$ be 
a $k$-bump solution given in Section 2. By Theorem 2.4, if $r$ is small enough and $l$ is large enough then for any $i \in\{1, \ldots, k\}$ either $\left.u\right|_{B_{R_{0}}\left(l j_{i}\right)}>0$ or $\left.u\right|_{B_{R_{0}}\left(l j_{i}\right)}<0$, and $\max |u|_{F_{l}^{c}} \mid<\delta$ where $F_{l}^{c}=\mathbf{R}^{N} \backslash F_{l}$. Then $\left.u\right|_{F_{l}}$ has $k$ nodal domains. We claim any point $x$ in $F_{l}^{c}$ for which $u(x) \neq 0$ has to be connected in $\left\{x \in \mathbf{R}^{N}: u(x) \neq 0\right\}$ to some nodal domains of $\left.u\right|_{F_{l}}$. If this is not the case, $\left.u\right|_{F_{l}^{c}}$ has a nodal domain $D$ which is not connected in $\left\{x \in \mathbf{R}^{N}: u(x) \neq 0\right\}$ to any of the nodal domains of $\left.u\right|_{F_{l}}$, therefore $D$ itself becomes a nodal domain of $u$. Since $u$ decays to 0 at infinity $u$ has a maximum or a minimum in $D$, for example, a maximum at $x_{0}$. But since $u\left(x_{0}\right)<\delta,\left|f\left(x_{0}, u\left(x_{0}\right)\right)\right| \leqslant \frac{V_{0}}{2} u\left(x_{0}\right)$, and $\Delta u\left(x_{0}\right) \leqslant 0$, we get a contradiction with the equation.

In case (ii), we have $\frac{\partial v}{\partial v}(x) \neq 0$ on $\partial \Omega$ and $\left|\frac{\partial v}{\partial v}(x)\right|$ has a positive lower bound on $B_{R_{0}(0)} \cap \partial \Omega$ for any $v \in A$, where $v$ is the outer unit normal to $\partial \Omega$. Again by Theorem 2.4, if $r$ is small enough and $l$ is large enough then for any $i \in\{1, \ldots, k\}$ either $\left.u\right|_{B_{R_{0}}}\left(l j_{i}\right) \cap \Omega>0$ or $\left.u\right|_{B_{R_{0}}\left(l j_{i}\right) \cap \Omega}<0$, and $\max |u|_{F_{l}^{c} \cap \Omega} \mid<\delta$. The rest is the same.

Theorem 1.1 follows from Lemma 4.1 right away.

Proof of Theorem 1.2. Let $k \geqslant m \geqslant 2$ and $k_{1}, k_{2}, \ldots, k_{m}$ be fixed positive integers such that $\sum_{s=1}^{m} k_{s}=k$. In this case the problem is $\mathbf{Z}$-invariant. Denote $i_{0}=0$ and $i_{n}=\sum_{s=1}^{n} k_{s}$ for $n \in\{1, \ldots, m\}$. We may assume in Theorem 2.2, $j_{1}<j_{2}<\cdots<j_{k}$ and we take $\lambda=\left(\lambda_{1}, \ldots, \lambda_{k}\right) \in \Lambda^{k}$ such that the first $k_{1}$ components are +1 , the next $k_{2}$ components are -1 , and keep taking alternating signs for $\lambda_{i}$ in this way. That is, $\lambda_{i}=(-1)^{n+1}$ for $i \in\left\{i_{n-1}+1, \ldots, i_{n}\right\}$ and $n \in\{1, \ldots, m\}$. We claim that with these choices the solutions given in Theorem 2.2 for sufficiently small $r$ and sufficiently large $l$ have exactly $m$ nodal domains $D_{1}, \ldots, D_{m}$ with $\left.u\right|_{D_{i}}$ being a positive (negative, resp.) $k_{i}$-bump solution for $i$ odd (even, resp.). First, there is $\delta>0$ such that $|f(x, t)| \leqslant \frac{V_{0}|t|}{2}$ for $|t| \leqslant \delta$. Then there is $R_{0}>0$ such that $v(x) \leqslant \delta / 2 k$ for all $v \in A$ and for $x \in \Omega \backslash \omega \times\left[-R_{0}, R_{0}\right]$. For any $v \in A, v(x)>0$ for $x \in \omega \times\left[-R_{0}, R_{0}\right]$ and $\frac{\partial v}{\partial v}(x)<0$ for $x \in \partial \Omega \cap\left(\mathbf{R}^{N-1} \times\left[-R_{0}, R_{0}\right]\right)$. By Theorem 2.4, if $r$ is small enough and $l$ is large enough then $|u(x)|<\delta$ for $x \in \Omega \backslash\left(\omega \times \bigcup_{i=1}^{k}\left[-R_{0}+l j_{i}, R_{0}+l j_{i}\right]\right)$ and $(-1)^{n+1} u(x)>0$ for $x \in \omega \times\left[-R_{0}+l j_{i}, R_{0}+l j_{i}\right]$ and for $i \in\left\{i_{n-1}+1, \ldots, i_{n}\right\}$ and $n \in\{1, \ldots, m\}$. Thus $u$ has at least $m$ nodal domains. To see that $u$ has exactly $m$ nodal domains, it suffices to prove that $(-1)^{n+1} u(x)>0$ for $x \in \omega \times$ $\left[-R_{0}+l j_{i_{n-1}+1}, R_{0}+l j_{i_{n}}\right]$ for all $n \in\{1, \ldots, m\}$ and that any nodal domain of $u$ contains one of the $m$ sets $\omega \times\left[-R_{0}+l j_{i_{n-1}+1}, R_{0}+l j_{i_{n}}\right]$. And it is in turn sufficient to prove that there is no nodal domain of $u$ contained completely in $\Omega \backslash\left(\omega \times \bigcup_{i=1}^{k}\left[-R_{0}+l j_{i}, R_{0}+l j_{i}\right]\right)$. Arguing indirectly, we assume that $\Omega^{*}$ is a nodal domain of $u$ which is contained completely in $\Omega \backslash\left(\omega \times \bigcup_{i=1}^{k}\left[-R_{0}+l j_{i}, R_{0}+l j_{i}\right]\right)$ and assume $\left.u\right|_{\Omega^{*}}>0$. Assume $\left.u\right|_{\Omega^{*}}$ attains its maximum at $x^{*}$. Since $\left.u\right|_{\Omega^{*}}<\delta$, we have $-\Delta u\left(x^{*}\right) \geqslant 0, V\left(x^{*}\right) u\left(x^{*}\right) \geqslant V_{0} u\left(x^{*}\right)$, and $f\left(x^{*}, u\left(x^{*}\right)\right)<\frac{V_{0}}{2} u\left(x^{*}\right)$, but this is a contradiction with the equation.

Proof of Theorem 1.3. Let $k \geqslant 2$ be fixed. In this case the problem again is $\mathbf{Z}$-invariant, we may assume in Theorem 2.3, $j_{1}<j_{2}<\cdots<j_{k}$ and we take $\lambda=\left(\lambda_{1}, \ldots, \lambda_{k}\right) \in \Lambda^{k}$ such that $\lambda_{i}$ takes alternating +1 and -1 with $\lambda_{1}=+1$, that is, $\lambda_{i}=+1$ for $i$ odd and $\lambda_{i}=-1$ for $i$ even. By Lemma 4.1, a solution $u$ given in Theorem 2.3 has at most $k$ nodal domains. We argue in the following that $u$ has at least $\left[\frac{k}{2}\right]+1$ nodal domains. Again, there is $R_{0}>0$ such that for $r$ small and $l$ large and for some $a_{0}>0, u(x)>a_{0}$ for $x \in B_{R_{0}}\left(l j_{i}\right)$ when $i$ is odd, $u(x)<-a_{0}$ for $x \in B_{R_{0}}\left(l j_{i}\right)$ when $i$ is even, and any point $x$ in $\mathbf{R}^{N}$ for which $u(x) \neq 0$ has to be connected in $\left\{x \in \mathbf{R}^{N}: u(x) \neq 0\right\}$ to one of the balls $B_{R_{0}}\left(l j_{i}\right)$. Here $l j_{i}$ is understood as $\left(0, \ldots, 0, l j_{i}\right)$. A useful observation here is that if $1 \leqslant i<n \leqslant k$ and $B_{R_{0}}\left(l j_{i}\right)$ and $B_{R_{0}}\left(l j_{n}\right)$ are subsets of one nodal domain in which $u$ is positive, then $B_{R_{0}}\left(l j_{p}\right)$ with $i<p<n$ and $B_{R_{0}}\left(l j_{q}\right)$ with $q<i$ or $q>n$ cannot be subsets of one nodal domain in which $u$ is negative. This is a consequence of the fact that $u$ is radially symmetric in the first $(N-1)$ variables. Similarly, if $1 \leqslant i<n \leqslant k$ and $B_{R_{0}}\left(l j_{i}\right)$ and $B_{R_{0}}\left(l j_{n}\right)$ are subsets of one nodal domain in which $u$ is negative, then $B_{R_{0}}\left(l j_{p}\right)$ with $i<p<n$ and $B_{R_{0}}\left(l j_{q}\right)$ with $q<i$ or $q>n$ cannot be subsets of one nodal domain in which $u$ is positive. Now, we use an induction procedure to prove the result. With our selected configurations of positive and negative bumps, we may identify the bump at $B_{R_{0}}\left(l j_{i}\right)$ to the integer $i$ so that positive bumps correspond to odd numbers and negative bumps correspond to even numbers. With this identification, two integers $i, n \in\{1, \ldots, k\}$ are said 
to be connected to each other if and only if $B_{R_{0}}\left(l j_{i}\right)$ and $B_{R_{0}}\left(l j_{n}\right)$ are subsets of one nodal domain of $u$. Then the number of nodal domains of $u$ is the number of connected components of one of the graphs formed by the $k$ integers $\{1,2, \ldots, k\}$ under the following rules: (a) odd numbers can only be connected to odd numbers, and even numbers can only be connected to even numbers; (b) if two odd numbers $i, n$ with $i<n$ are connected to each other then any even number inside the interval $[i, n]$ cannot be connected to any even number outside that interval; (c) switch the roles of odd numbers and even numbers in (b). To get the result, it is sufficient to prove that $\left[\frac{k}{2}\right]+1$ is a lower bound of the numbers of connected components of the graphs formed from $\{1, \ldots, k\}$ by the above rules. This is obvious for $k=1,2,3$ by a direct inspection. Assume the claim is true for some positive integer $k$ and consider the graph formed from the set $\{1, \ldots, k+1\}$. If $k+1$ is not connected to the proceeding numbers $1, \ldots, k$ then by the induction assumption the number of connected components of the graph is at least $\left[\frac{k}{2}\right]+1+1$ which is not less than $\left[\frac{k+1}{2}\right]+1$. If $k+1$ is connected to some $j \in\{1, \ldots, k\}$ then any integer in the set $\{j+1, \ldots, k\}$ which is connected to an integer less than $j$ must be in the component containing $j$ and $k+1$. Therefore, when the number of connected components containing integers in the set $\{1, \ldots, j\}$ is considered, one may regard the set $\{j, \ldots, k+1\}$ as one integer $j$ and ignore the integers $j+1, \ldots, k+1$ and by using the induction assumption, we know there are at least $\left[\frac{j}{2}\right]+1$ connected components containing integers in $\{1, \ldots, j\}$. This means there are at least $\left[\frac{j}{2}\right]$ connected components which do not contain any integers in $\{j, \ldots, k+1\}$. Also, when the number of connected components containing integers in $\{j+1, \ldots, k+1\}$ is considered, one may ignore the integers $\{1, \ldots, j\}$ since any integer in $\{1, \ldots, j\}$ connected to an integer in $\{j+1, \ldots, k+1\}$ must be connected to $k+1$. By the induction assumption again there are at least $\left[\frac{k+1-j}{2}\right]+1$ connected components which contain integers in $\{j+1, \ldots, k+1\}$, or equivalently, which contain integers in $\{j, \ldots, k+1\}$. The total number of connected components is the sum of the number of connected components which do not contain any integers in $\{j, \ldots, k+1\}$ and the number of connected components which contain integers in $\{j, \ldots, k+1\}$. Therefore, the total number of connected components of the graph formed by $\{1, \ldots, k+1\}$ is at least $\left[\frac{j}{2}\right]+\left[\frac{k+1-j}{2}\right]+1$. Since $k+1$ is connected to $j, k+1$ and $j$ must be either both even numbers or both odd numbers. In either case $k+1-j$ is an even number, which implies $\left[\frac{j}{2}\right]+\left[\frac{k+1-j}{2}\right]=\left[\frac{k+1}{2}\right]$. This finishes the induction process. Going back to the original problem we find that $u$ has at least $\left[\frac{k}{2}\right]+1$ nodal domains.

\section{Acknowledgements}

The authors are grateful to the referee for his inspiring comments which led to Remark 2.5 and to Paul Rabinowitz for his encouragements of this work. This paper was written when the first author was visiting Utah State University. He is grateful to the members in the Department of Mathematics and Statistics at Utah State University for their invitation and hospitality.

\section{References}

[1] N. Ackermann, T. Weth, Multibump solutions to nonlinear periodic Schrödinger equations in a degenerate setting, Comm. Contemp. Math., submitted for publication.

[2] T. Bartsch, Critical point theory on partially ordered Hilbert spaces, J. Funct. Anal. 186 (2001) 117-152.

[3] T. Bartsch, K.C. Chang, Z.-Q. Wang, On the Morse indices of sign-changing solutions for nonlinear elliptic problems, Math. Z. 233 (2000) 655-677.

[4] T. Bartsch, Z.L. Liu, T. Weth, Sign changing solutions of superlinear Schrödinger equations, Comm. Partial Differential Equations 29 (2004) 25-42.

[5] T. Bartsch, Z.-Q. Wang, On the existence of sign changing solutions for semilinear Dirichlet problems, Topol. Methods Nonlinear Anal. 7 (1996) 115-131.

[6] H. Brezis, L. Nirenberg, $H^{1}$ versus $C^{1}$ local minimizers, C. R. Acad. Sci. Paris Sér. I Math. 317 (1993) 465-472.

[7] A. Castro, J. Cossio, J. Neuberger, A sign-changing solution for a superlinear Dirichlet problem, Rocky Mountain J. Math. 27 (1997) 1041-1053. 
[8] K.C. Chang, Infinite Dimensional Morse Theory and Multiple Solution Problems, Progr. Nonlinear Differential Equations Appl., vol. 6, Birkhäuser, Boston, 1993

[9] K.C. Chang, $H^{1}$ versus $C^{1}$ isolated critical points, C. R. Acad. Sci. Paris Sér. I Math. 319 (1994) 441-446.

[10] V. Coti Zelati, P.H. Rabinowitz, Homoclinic orbits for second order Hamiltonian systems possessing superquadratic potentials, J. Amer. Math. Soc. 4 (1991) 627-693.

[11] V. Coti Zelati, P.H. Rabinowitz, Homoclinic type solutions for a semilinear elliptic PDE on $\mathbf{R}^{n}$, Comm. Pure Appl. Math. 45 (1992) $1217-1269$.

[12] E.N. Dancer, Y. Du, On sign-changing solutions of certain semilinear elliptic problems, Appl. Anal. 56 (1995) $193-206$.

[13] E.N. Dancer, S. Yan, A singularly perturbed elliptic problem in bounded domains with nontrivial topology, Adv. Differential Equations 4 (1999) 347-368.

[14] D. Gilbarg, N.S. Trudinger, Elliptic Partial Differential Equations of the Second Order, Springer-Verlag, Berlin, 1983.

[15] S.J. Li, Z.-Q. Wang, Mountain pass theorem in order intervals and multiple solutions for semilinear elliptic Dirichlet problems, J. Analyse Math. 81 (2000) 373-396.

[16] S.J. Li, Z.-Q. Wang, Ljusternik-Schnirelman theory in partially ordered Hilbert spaces, Trans. Amer. Math. Soc. 354 (2002) $3207-3227$.

[17] Z.L. Liu, J.X. Sun, Invariant sets of descending flow in critical point theory with applications to nonlinear differential equations, J. Differential Equations 172 (2001) 257-299.

[18] Z.L. Liu, Z.-Q. Wang, Multi-bump type nodal solutions having a prescribed number of nodal domains: II, Ann. I. H. Poincaré - AN 22 (2005) 609-631.

[19] P.H. Rabinowitz, Minimax Methods in Critical Point Theory with Applications to Differential Equations, CBMS Regional Conf. Ser. in Math., vol. 65, Amer. Math. Soc., Providence, RI, 1986.

[20] P.H. Rabinowitz, A variational approach to multibump solutions of differential equations, in: Contemp. Math., vol. 198, Amer. Math. Soc., Providence, RI, 1996, pp. 31-43.

[21] P.H. Rabinowitz, Multibump solutions of differential equations: an overview, Chinese J. Math. 24 (1996) 1-36.

[22] E. Séré, Existence of infinitely many homoclinic orbits in Hamiltonian systems, Math. Z. 209 (1992) $27-42$. 